\title{
Performance of gentamicin population kinetic parameters in Portuguese neonates
}

\author{
Marília J. Rocha • Anabela M. Almeida • \\ Amílcar C. Falcão • Margarida M. Caramona
}

Received: 7 September 2004 / Accepted: 2 October 2005/Published online: 13 March 2007

(C) Springer Science + Business Media B.V. 2007

\begin{abstract}
Aim: To evaluate the performance of eight different sets of gentamicin populational pharmacokinetic parameters, regarding potential implementation in clinical pharmacokinetic software as prior information.

Methods: The study involved 49 patients of $31.3 \pm 4.1$ weeks of gestational age (GA), receiving gentamicin, and for whom peak and trough concentrations were obtained. Accuracy and precision were assessed by mean prediction error (ME), mean squared prediction error (MSE) and root mean squared prediction error (RMSE). Weighted prediction-error analysis was carried out in order to evaluate peak and trough concentrations together $\left(\mathrm{ME}_{\mathrm{w}}, \mathrm{MSE}_{\mathrm{w}}\right.$ and $\left.\mathrm{RMSE}_{\mathrm{w}}\right)$. Results: The analysis showed $\mathrm{CL}=0.036 \mathrm{1} / \mathrm{h} / \mathrm{kg}$ $(<34$ weeks GA) or $\mathrm{CL}=0.051 \mathrm{l} / \mathrm{h} / \mathrm{kg} \quad(\geq 34$ weeks GA), and $V_{d}=0.51 / \mathrm{kg}\left(\leq 37\right.$ weeks GA) or $V_{d}=0.41 / \mathrm{kg}$ $(>37$ weeks of GA) as the most accurate and precise set of pharmacokinetic parameters (Set 4), presenting the highest percentage of clinically acceptable estimates (Error $_{\text {Peak }}<1 \mu \mathrm{g} / \mathrm{ml}$, and Error Trough $<0.375 \mu \mathrm{g} / \mathrm{ml}$ ). Conclusion: The adoption of the previously mentioned set of parameters as population estimates seems to be the best option, bearing in mind the obtained results. However, we strongly believe that pharmacokinetic parameter determination of gentamicin should be carried out whenever possible in order to improve the rationale and cost-effectiveness of therapy.
\end{abstract}

Key words Gentamicin · Infant · Newborn · Pharmacokinetics $\cdot$ Prediction error

M. J. Rocha · A. M. Almeida

A. C. Falcão $(\bowtie) \cdot$ M. M. Caramona

Laboratory of Pharmacology, Faculty of Pharmacy, University of Coimbra, 3000-295, Coimbra, Portugal

E-mail: acfalcao@ff.uc.pt

\section{Introduction}

Intensive care unit (ICU) patients routinely meet the criteria outlined by several authors to be included in therapeutic drug monitoring programmes. Dosage adjustments based on individual pharmacokinetic parameters are of considerable importance for effective and safe use of drugs [1,2]. This is especially true for children, for whom the kinetic and dynamic processes are usually not as well characterised as for the adult population. Neonates present the additional challenge of having a set of mechanisms changing with alarming rapidity on a day-to-day basis [3-5].

In reviewing paediatric literature, there is an obvious lack of information for most therapeutic agents in neonates concerning pharmacokinetic and/or pharmacodynamic data. Furthermore, some of the most often used drugs in this subpopulation (e.g., antibiotics) present relatively narrow therapeutic ranges. Gentamicin, which is often used in the treatment of suspected or proven gram-negative infections in this age group, has been shown to be ototoxic and nephrotoxic in the adult population. Although gentamicin toxicity in newborns is not well documented, it may be reasonable to expect similar or even more pronounced effects in infants [1, 4].

In order to improve gentamicin therapy in Portuguese neonates, the Bayesian approach seems to be an appropriate methodology to optimise the dosage schedules required to achieve desirable target concentrations (peak and trough serum levels).
Aim
The aim of the present work was to evaluate the per- formance of eight different sets of gentamicin phar- macokinetic parameters, obtained by different authors, regarding potential implementation in a clinical phar- macokinetic program (PKS System; Abbott Labora- tories, Abbott Park, IL, USA) as prior information. 


\section{Methods}

\section{Patients}

A retrospective review of pharmacokinetic data of gentamicin was performed in 49 neonates admitted in the neonatal intensive care unit at Coimbra University Hospital (CUH) between 2000 and 2003. All the selected patients had a postnatal age of below one week and two available gentamicin serum concentrations (peak and trough) obtained according to routine clinical protocol. The group had the following characteristics, expressed as mean and standard deviation: birth weight $(1.79 \pm 0.80 \mathrm{~kg}), \quad$ gestational age $(31.3 \pm$ 4.1 weeks $)$, and serum creatinine $(0.96 \pm 0.30 \mathrm{mg} / \mathrm{dl})$.

\section{Gentamicin dosing and sampling times}

A standard $2.5 \mathrm{mg} / \mathrm{kg}$ dose the gentamicin was administered through a 10 -min slow intravenous infusion and the interval between doses was selected on the basis of patients' weight: 24-, 18- and 12-h intervals for weights $<1200,1200-2000$ and $>2000 \mathrm{~g}$, respectively.

Two blood samples ( $1 \mathrm{~h}$ after the end of administration, and $30 \mathrm{~min}$ before the next dose) were routinely collected after 24 and $48 \mathrm{~h}$ starting the therapy. The assumed target concentrations for gentamicin were defined to be $0.5-2$ and $6-10 \mu \mathrm{g} / \mathrm{ml}$ for trough and peak levels, respectively $[2,3,6]$.

Serum samples were collected via venipuncture and gentamicin concentrations were determined by a fluorescence polarisation immunoassay technique (TDx; Abbott Diagnostics) at the Laboratory of Hormonology and Therapeutic Drug Monitoring of CUH. This assay had a coefficient of variation (CV) lower than $6 \%$ in our institution and the sensitivity was $0.27 \mu \mathrm{g} /$ $\mathrm{ml}$; these two values were taken into account for subsequent analysis.

\section{Pharmacokinetic analysis}

The PKS computer program (Abbott Laboratories, Abbott Park, IL, USA) was used to evaluate the prediction of gentamicin concentrations in our population. The kinetic analysis was carried out assuming a one-compartment open model with zero-order absorption (short infusion) and first-order elimination. Since no database concerning gentamicin information in a newborn population was available in the original program package, the populational pharmacokinetic parameters used for estimating the predictive performance were those most reported in the literature. Several sets of gentamicin pharmacokinetic parameters involving populational estimates for both gentamicin clearance and volume of distribution were established according to different authors (Table 1). Eight Sets were then tested: four (Sets 1-4) were obtained using traditional methodology (Stand TwoStage Method - STS), and the remaining four (Sets 5-8) were determined through an alternative methodology (Populational Approach - PA). These Sets were used to calculate the predicted concentrations according to the demographic and clinical characteristics of the patients, which provided the present work with the clinical environment for this kind of population research.

\section{Predictive performance}

The absolute and relative predictive performances were evaluated applying the Prediction-Error analysis as suggested by Sheiner \& Beal [9] the most used methodology involving comparison of predicted (calculated by the Sets) and observed (measured) drug concentrations. Prediction error was defined as the predicted minus the observed serum gentamicin concentrations. The Prediction-Error analysis involved the calculation of mean prediction error (ME), a measure of accuracy; mean squared prediction error (MSE), representing precision; and the root mean squared prediction error (RMSE), a measure of both accuracy and precision. Weighted (normalisation of the ME by the observed concentrations) prediction-error analysis $\left(\mathrm{ME}_{\mathrm{w}}, \mathrm{MSE}_{\mathrm{w}}\right.$ and $\mathrm{RMSE}_{\mathrm{w}}$ ) was carried out in order to evaluate all (peak and trough) concentrations together.

The smaller the ME, MSE and RMSE, the less biased and more precise the results should be. Bias was tested by comparing the mean prediction error with zero using the Student's $t$-test. Comparison of the predictive capacity of the different Sets, regarding accuracy and precision, was done by analysis of variance (ANOVA). Relative predictive performance was assessed by the differences in values for $\mathrm{ME}(\triangle \mathrm{ME})$ and MSE ( $\triangle \mathrm{MSE})$ and their $95 \%$ confidence intervals. If the $95 \%$ confidence intervals for the relative performance criteria did not include zero, the predictions were considered to be significantly different. Parametric statistical calculations were used because the data were normally distributed. The significant probability level chosen was $P \leq 0.05$. Statistica ${ }^{\circledR}$ for Windows (Version 6.1; Statsoft, Inc., Tulsa, OK, USA) was used to perform all statistical tests.

To assess the practical clinical performance of our sets, the individual prediction errors were arranged in two groups for peak and trough concentrations [10]: errors higher than $1 \mu \mathrm{g} / \mathrm{ml}$ for peak and $0.375 \mu \mathrm{g} / \mathrm{ml}$ for trough serum levels were considered as unacceptable errors. The clinical performance of each set of parameters was evaluated according to the percentage of acceptable estimates. 
Table 1 Population pharmacokinetic parameters of gentamicin

\begin{tabular}{|c|c|c|c|c|}
\hline & & \multicolumn{2}{|l|}{ Parameters } & \multirow[t]{2}{*}{ References } \\
\hline & & $\mathrm{CL}(\mathrm{l} / \mathrm{h} / \mathrm{kg})$ & $V_{d}(1 / \mathrm{kg})$ & \\
\hline \multirow[t]{4}{*}{ Set 1} & GA $23-27$ & 0.052 & 0.540 & \multirow[t]{4}{*}{ Murphy et al. [3] } \\
\hline & GA $28-33$ & 0.043 & 0.480 & \\
\hline & GA $34-37$ & 0.047 & 0.460 & \\
\hline & GA $38-42$ & 0.047 & 0.410 & \\
\hline \multirow[t]{4}{*}{ Set 2} & GA $<28$ & 0.041 & 0.610 & \multirow{4}{*}{ Semchuk et al. [6] } \\
\hline & GA $28-29$ & 0.045 & 0.660 & \\
\hline & GA $30-34$ & 0.048 & 0.530 & \\
\hline & GA $34-40$ & 0.081 & 0.510 & \\
\hline \multirow[t]{2}{*}{ Set 3} & $\mathrm{GA} \leq 34$ & 0.043 & 0.670 & \multirow{2}{*}{ Semchuk et al. [4] } \\
\hline & $\mathrm{GA}>34$ & 0.048 & 0.520 & \\
\hline \multirow[t]{3}{*}{ Set 4} & GA $<34$ & 0.036 & 0.500 & \multirow[t]{3}{*}{ Delgado et al. [2] } \\
\hline & GA $34-37$ & 0.051 & 0.500 & \\
\hline & $\mathrm{GA}>37$ & 0.051 & 0.400 & \\
\hline \multirow[t]{2}{*}{ Set 5} & \multicolumn{3}{|c|}{$\begin{array}{l}\text { Covariates for CL (1/h): WT (kg); GA (weeks); PNA (days); PCA } \\
\text { (weeks) }\end{array}$} & \multirow[t]{2}{*}{ Izquierdo et al. [7] } \\
\hline & \multicolumn{3}{|c|}{ Covariate for $V_{d}(\mathrm{l})$ : WT $(\mathrm{kg})$} & \\
\hline \multirow[t]{2}{*}{ Set 6} & \multicolumn{3}{|c|}{$\begin{array}{l}\begin{array}{l}\text { Covariates for } \mathrm{CL}(\mathrm{l} / \mathrm{h}) \text { : WT }(\mathrm{kg}) \text {; Age (days); Serum creatinine } \\
(\mu \mathrm{mol} / \mathrm{l})\end{array}\end{array}$} & \multirow[t]{2}{*}{ Kelman et al. [5] } \\
\hline & \multicolumn{3}{|c|}{ Covariate for $V_{d}(\mathrm{l})$ : WT $(\mathrm{kg})$} & \\
\hline \multirow[t]{2}{*}{ Set 7} & \multicolumn{3}{|c|}{ Covariates for CL (1/h): WT (kg); PCA (weeks); Apgar } & \multirow[t]{2}{*}{ Thomson et al. [8] } \\
\hline & \multicolumn{3}{|c|}{ Covariate for $V_{d}(\mathrm{l})$ : WT $(\mathrm{kg})$} & \\
\hline \multirow[t]{2}{*}{ Set 8} & \multicolumn{3}{|c|}{$\begin{array}{l}\text { Covariates for CL }(1 / \mathrm{h}) \text { : WT }(\mathrm{kg}) \text {; GA (weeks); Serum creatinine } \\
(\mu \mathrm{mol} / \mathrm{l})\end{array}$} & \multirow[t]{2}{*}{ Weber et al. [1] } \\
\hline & \multicolumn{3}{|c|}{ Covariate for $V_{d}(\mathrm{~L}): \mathrm{WT}(\mathrm{kg})$} & \\
\hline
\end{tabular}

WT, weight; GA, gestational age; PCA, postconceptional; PNA, postnatal age. Sets 1-4: Standard Two-Stage (STS); Sets 5-8: Population Approach (PA).

\section{Results}

The predictive performances of the eight Sets were evaluated by prior prediction for peak and trough levels. It must be emphasised that all sets presented negative ME values for both peak and trough levels, indicating an underprediction of the observed concentrations. In the STS group (Sets 1-4), Set 4 exhibited the best accuracy and precision measures evaluated by ME, MSE, and confirmed by RMSE values for peak concentrations. This is also true for trough levels, except for accuracy, for which the predictive capacity of Sets 3 and 4 appears to be similar. In the PA group (Sets 5-8), Set 7 showed the best accuracy and precision measures for peak levels. Otherwise, concerning trough concentrations, Set 8 appears to be slightly better than Set 7. However, when the available data (peak and trough levels) were analysed together using the weighted absolute predictive capacity $\left(\mathrm{ME}_{\mathrm{w}}, \mathrm{MSE}_{\mathrm{w}}\right.$ and $\left.\mathrm{RMSE}_{\mathrm{w}}\right)$, Sets 4 and 7 proved to be the best sets of pharmacokinetic parameters regarding the predictive performance (Table 2).

Additionally, Sets 4 and 7 were selected as a reference in order to evaluate the relative predictive capacity of Sets in the STS group and PA group, respectively. All Sets denoted significant differences when compared with the references for both accuracy and precision. Furthermore, the comparison between Sets 4 and 7 revealed Set 4 as the most precise and accurate set of pharmacokinetic parameters. These observations were confirmed by the variance analysis performed to find differences between the Sets.

Finally, the clinical performance of the methods was evaluated by the percentage of acceptable errors (Error $_{\text {Peak }}<1$ and Error $_{\text {Trough }}<0.375 \mu \mathrm{g} / \mathrm{ml}$ ). Set 4 showed the largest percentage $(34 \%)$ of clinically acceptable errors in prior prediction; on the other hand, Set 8 expressed poor precision and accuracy, showing the highest percentage $(84 \%)$ of rejected estimate concentrations, which is a very high value considering its potential application in clinical practice. 


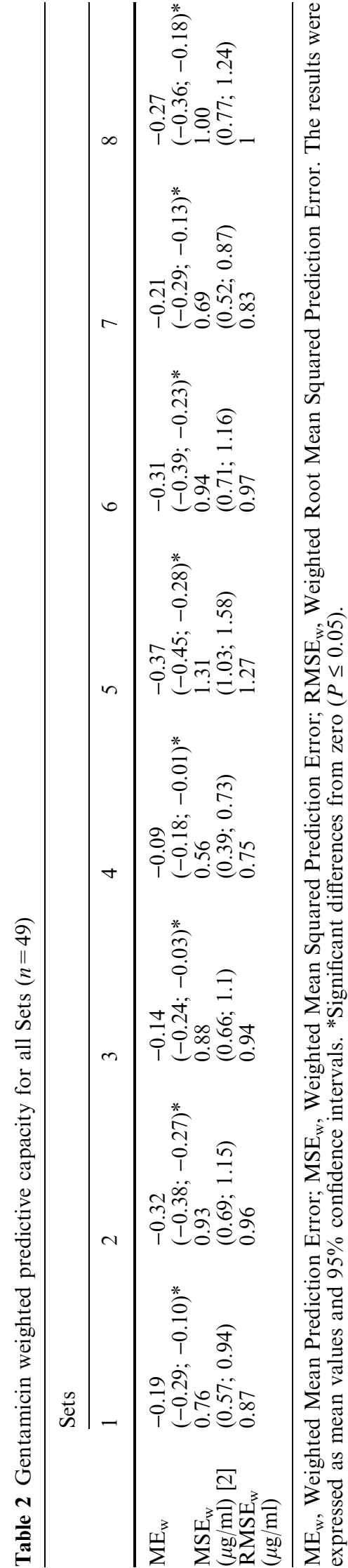

\section{Discussion}

The variability in the pharmacodynamic response following administration of standard doses is due in part to inter- and intra-individual pharmacokinetic variability. Furthermore, the effects of drugs on developmental processes that are on going in preterm and term neonates cannot be assessed in older children or adults $[2,3,7]$.

Bearing in mind the wide range of values for the populational pharmacokinetic parameters of gentamicin presented by the selected bibliographical information (Table 1) and the constant evolution of children, it seemed important to analyse their predictive performance in order to characterise our population kinetic profile. In fact, investigations into the effects on drug disposition in these patients have been made with the aim of providing improved a priori predictions of serum concentration time profiles and thereby dynamic response. Despite these advances in theory and practice, the accuracy with which drug dosage regimens and/or the likelihood of drug interactions can be predicted for the individual patient remains problematic $[3,5,7)$.

In the present work, from the prediction-error analysis of the eight sets of pharmacokinetic parameters obtained from the literature, it must be emphasised that all of them presented negative ME values for both peak and trough levels, indicating a persistent underprediction of the observed concentrations. This may be related to the specific characteristics of our study population, $39 \%$ of which consisted of neonates of 26-28 weeks of gestational age.

In the STS group (Sets 1-4), Method 4 [2] was the most similar concerning demographic and clinical characteristics of the studied neonates, which may explain the acceptable clinical performance showed by this set of pharmacokinetic parameters regarding gentamicin concentration predictions. Additionally, it must be pointed out that the mentioned study was carried out in Spain, which is the nearest country to Portugal in terms of geographic, demographic and cultural aspects. In the PA group (Sets 5-8), Set 7 [8] appeared to be the best one in terms of predictive capacity, which can be confirmed by the clinically acceptable errors in prior prediction. Although Set 5 [7] was carried out with Spanish neonates (as happened with Set 4), the studied population did not include patients with low gestational age, which can explain the relatively poor clinical performance of this set of pharmacokinetic parameters in comparison with Set 7.

This analysis sustained as an other studies a significant portion of the variability in gentamicin pharmacokinetics parameters in neonates less 7 days old and, unlike current step-based approaches, allows the gentamicin dose to reflect the difference in gestational age within each dosing group [1, 4]. 


\section{Conclusion}

The obtained results led us to choose Sets 4 and 7 as the most reliable sets of parameters for the STS group and PA group, respectively. Furthermore, a comparison between both methods revealed that Set 4 $(\mathrm{CL}=0.036 / 0.051 \mathrm{l} / \mathrm{h} / \mathrm{kg}$ for neonates of gestational age $<34$ or $\geq 34$ weeks, respectively; $V_{d}=0.5 / 0.41 / \mathrm{kg}$ for neonates of gestational age $\leq 37$ or $>37$ weeks, respectively) is the most accurate and precise in predicting gentamicin concentrations. Otherwise, it must be stressed that the adopted methodology for the determination of populational pharmacokinetic parameters (STS versus PA) did not appear to be decisive for the accuracy and precision of gentamicin predictions, but caution must be exercised in extrapolating kinetic information to patients in other settings without previous validation.

As a final remark, despite the acceptable predictive and clinical performances obtained with Set 4, the overall results of our study support the idea that neonate gentamicin pharmacokinetic parameters in our population should be determined in order to improve the rationale and cost-effectiveness of this antimicrobial therapy.

\section{References}

1. Weber W, Kewitz G, Rost KL, Lobby M, Nitz M, Harnisch L. Population kinetics of gentamicin in neonates. Eur J Clin Pharmacol 1993; 44(Suppl. 4): S23-S25
2. Delgado RG, Romero AS, Gil RT, Macián AM. Monitorizacón de niveles séricos de gentamicina en neonatos. Utilidade para el ajuste de dosis. Anales Españoles de Pediatria 1997; 46:47-52

3. Murphy JE, Austin ML, Frye R. Evaluation of gentamicin pharmacokinetics and dosing protocols in 195 neonates. Am J Health-Syst Pharm 1998; 55:2280-2288

4. Semchuk W, Shevchuk YM, Sankaran K, Wallace SM. Prospective, randomized, controlled evaluation of a gentamicin loading dose in neonates. Biol Neonate 1995; 67: $13-20$

5. Kelman AW, Thomson AH, Whiting B, Bryson SM, Steedman DA, Mawer GE et al. Estimation of gentamicin and volume of distribution in neonates and young children. B J Clin Pharmcol 1984; 18:685-692

6. Semchuk W, Borgmann J, Bowman L. Determination of a gentamicin loading dose in neonates and infants. Ther Drug Monitor 1993; 15:47-51

7. Izquierdo $\mathrm{M}$, Lanao JM, Cervero L, Jimenez NV, Dominguez-Gil A. Population pharmacokinetics of gentamicin in premature infants. Ther Drug Monitor 1992; $14: 177-183$

8. Thomson AH, Way S, Bryson SM, McGovern EM, Kelman AW, Whiting B. Population pharmacokinetics of gentamicin in neonates. Dev Pharmacol Ther 1988; 11:173179

9. Sheiner LB, Beal SL. Some suggestions for measuring predictive performance. J Pharmacokinet Biopharm 1981; 4:503-512

10. Faura CC, Feret MA, Horga JF. Monitoring serum levels of gentamicin to develop a regimen for gentamicin dosage in newborns. Ther Drug Monitor 1991; 13:268-276 